\title{
Identification of the first oomycete mating-type locus sequence in the grapevine downy mildew pathogen, Plasmopara viticola
}

Yann Dussert $^{1 \dagger *}$, Ludovic Legrand ${ }^{2}$, Isabelle D. Mazet ${ }^{1}$, Carole Couture $^{1}$, Marie-Christine Piron ${ }^{3}$, Rémy-Félix Serre ${ }^{4}$, Olivier Bouchez ${ }^{4}$, Pere Mestre $^{3}$, Silvia Laura Toffolatti ${ }^{5}$, Tatiana Giraud ${ }^{6}$, François Delmotte ${ }^{1}$

${ }^{1}$ INRAE, Bordeaux Sciences Agro, Université Bordeaux, SAVE, F-33140, Villenave d'Ornon, France

${ }^{2}$ LIPM, INRAE, Université de Toulouse, CNRS, Castanet-Tolosan, France

${ }^{3}$ Université de Strasbourg, INRAE, SVQV UMR-A 1131, F-68000 Colmar, France

${ }^{4}$ INRAE, US 1426, GeT-PlaGe, Genotoul, Castanet-Tolosan, France

${ }^{5}$ Dipartimento di Scienze Agrarie e Ambientali, Università degli Studi di Milano, Milano, Italy

${ }^{6}$ Ecologie Systematique et Evolution, CNRS, AgroParisTech, Universite Paris-Saclay, 91400

Orsay France

${ }^{\dagger}$ Current affiliation: School of Biological and Chemical Sciences, Queen Mary University of London, London, UK

*Author for correspondence: Yann Dussert, dussert.yann@gmail.com 


\begin{abstract}
Mating types are self-incompatibility systems that promote outcrossing in plants, fungi and oomycetes. Mating-type genes have been widely studied in plants and fungi, but have yet to be identified in oomycetes, eukaryotic organisms closely related to brown algae that cause many destructive animal and plant diseases. We identified the mating-type locus of Plasmopara viticola, the oomycete responsible for grapevine downy mildew, one of the most damaging grapevine diseases worldwide. Using a genome-wide association approach, we identified a 570 $\mathrm{kb}$ repeat-rich non-recombining region controlling mating types, with two highly divergent alleles. We showed that one mating type was homozygous, whereas the other was heterozygous at this locus. The mating-type locus encompassed 40 genes, including one encoding a putative hormone receptor. Our findings have fundamental implications for our understanding of the evolution of mating types, as they reveal a unique determinism involving an asymmetry of heterozygosity, as in sex chromosomes and unlike other mating-type systems. This identification of the mating-type locus in such an economically important crop pathogen also has applied implications, as outcrossing facilitates rapid evolution and resistance to harsh environmental conditions.
\end{abstract}

Keywords: self-incompatibility system, mating-type, oomycetes, stramenopiles 


\section{INTRODUCTION}

Mating systems control the degree of outcrossing in natural populations, thus affecting adaptability (Lande \& Schemske 1985; Charlesworth \& Charlesworth 1987; Charlesworth et al. 1990; Igic et al. 2008; Lande \& Porcher 2015). Indeed, outcrossing promotes gene flow and, therefore, the rapid spread of beneficial alleles, the generation of new allelic combinations and the purging of deleterious alleles. Various genetic systems have evolved across the tree of life to enforce outcrossing, such as separate sexes or mating types (Beukeboom \& Perrin 2014). The sex of many plants and animals is determined by sex chromosomes (Beukeboom \& Perrin 2014). Mating types, preventing mating between individuals carrying the same alleles, have evolved independently in many lineages of the tree of life, including fungi, ciliates, green algae and oomycetes (Billiard et al. 2012; Sekimoto 2017; Orias et al. 2017). Plants possess a molecular system called self-incompatibility, which enforces outcrossing and is analogous to mating type (Fujii et al. 2016). Given the fundamental importance of mating types in life cycles and evolution, their molecular determinism has been extensively studied, particularly in plants, fungi and ciliates. Mating types are controlled by a number of different mechanisms, even within these groups (Billiard et al. 2012; Fujii et al. 2016; Orias et al. 2017).

Mating type systems enforcing outcrossing at the diploid stage, involving only two mating types, have been described in oomycetes on the basis of cross incompatibilities (Judelson 2009). However, no genomic sequence for a mating-type locus has yet been identified in this lineage. Oomycetes are diploid eukaryotic organisms closely related to diatoms and brown algae (Lévesque 2011). This group includes a number of animal and plant pathogens causing significant environmental and economic damage. Oomycetes are responsible for damaging diseases, such as saprolegniosis, a lethal disease affecting wild and farmed fish (van West 2006), sudden oak death and downy mildew in several crops (e.g., Bremia lactucae, responsible for 
lettuce downy mildew, and Plasmopara halstedii, responsible for sunflower downy mildew), and the infamous potato blight caused by Phytophthora infestans, responsible for the Irish potato famine in the 1840 s.

Mating types are of the utmost importance in these organisms, as they control gamete compatibility, and sexual reproduction generates thick-walled spores called oospores that can resist harsh conditions and survive for several years. Outcrossing also produces recombinant genotypes, which can mediate faster adaptation to control measures. For example, invasive populations of $P h$. infestans were not able to reproduce sexually for a long period, due to the lack of one mating type, but the recent introduction of the missing mating type has resulted in higher genetic diversity in some areas (Brurberg et al. 2011), and the emergence of an aggressive lineage (Gavino et al. 2000). In oomycetes, the mating-type locus has been located only on a genetic linkage map in Phytophthora species (Judelson et al. 1995; Fabritius \& Judelson 1997; Van Der Lee et al. 2004) and Bremia lactucae (Sicard et al. 2003), and the only known matingtype factors are purified hormones in Phytophthora spp. (Qi et al. 2005; Harutyunyan et al. 2008; Ojika et al. 2011). However, no genes determining mating type have yet been identified in oomycetes.

Plasmopara viticola, a pathogen causing grapevine downy mildew, one of the most devastating grapevine diseases worldwide, is an oomycete. Plasmopara viticola was introduced into European vineyards from North America in the 1870s (reviewed in Fontaine et al. 2013) and rapidly spread, invading vineyards all over the world. The life cycle of $P l$. viticola includes an asexual multiplication phase during the spring and summer and a sexual reproduction event in the fall, generating the thick-walled sexual spores (oospores) required for overwintering (Vercesi et al. 2010). In Pl. viticola, only two mating types have been observed, and mating can occur only between diploid individuals of different mating types (Wong et al. 2001). This results in high 
rates of outcrossing, a key element explaining rapid adaptation to fungicides (Toffolatti et al. 2011; Delmas et al. 2017) and to resistant cultivars (Peressotti et al. 2010; Delmas et al. 2016) in this species. We identified the first oomycete mating-type locus sequence, with an approach combining phenotypic analysis (mating types determined by crosses) with genome-wide singlenucleotide polymorphisms (SNPs) in Pl. viticola.

\section{RESULTS}

We determined the mating type of 54 diploid individuals of $P l$. viticola collected across Europe (Supplementary Table 1), in experimental crosses against six testers, three for each of the two mating types, arbitrarily called P1 and P2. The P1 mating type was inferred for an individual if it produced oospores when inoculated on grapevine leaves with P2 testers but not with P1 testers, and conversely for P2. We identified 26 individuals of the P1 mating type and 28 of the P2 mating type (Supplementary Table 2). We sequenced the genomes of these 54 diploid individuals with short-read technology, and mapped the reads onto a recent high-quality reference sequence obtained by long-read sequencing with high coverage (Dussert et al. 2019). We retained 2.011 million SNPs after filtering. No genetic subdivision associated with mating types was detected in population structure analyses based on principal component analysis or clustering analysis applied to a dataset filtered for SNPs in close linkage disequilibrium (LD) (Supplementary Figure 1). Conditions were therefore favorable for genetic-phenotype association studies.

Using a genome-wide association approach, we identified two genomic regions with significant signals of association with the mating-type phenotype, located at the edges of the scaffolds Plvit020 and Plvit030 (Figure 1). SNPs at these two scaffold extremities were in very strong LD (Figure 1B), indicating that they were located in the same genomic region and that this 
A

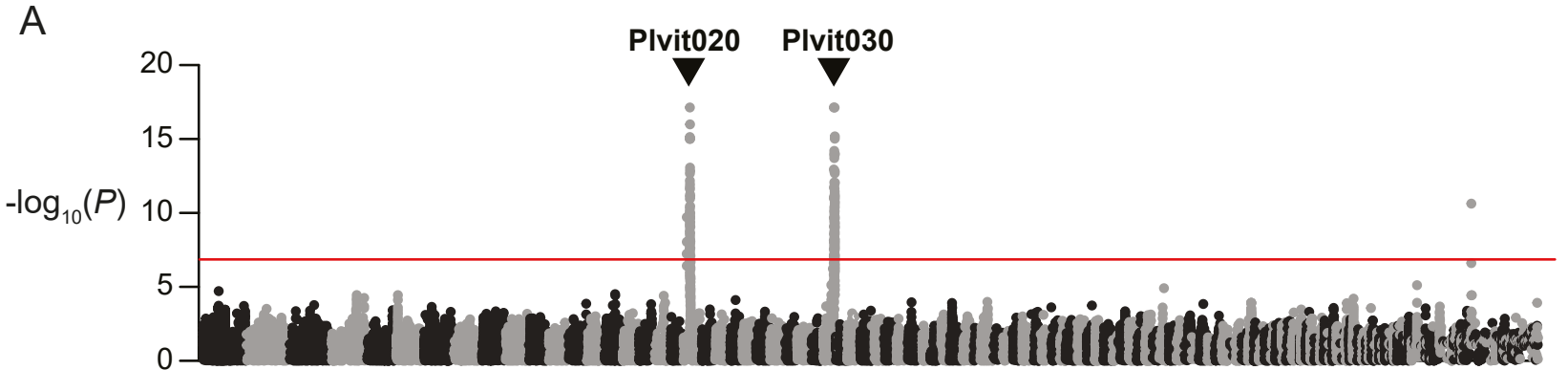

Scaffolds

B

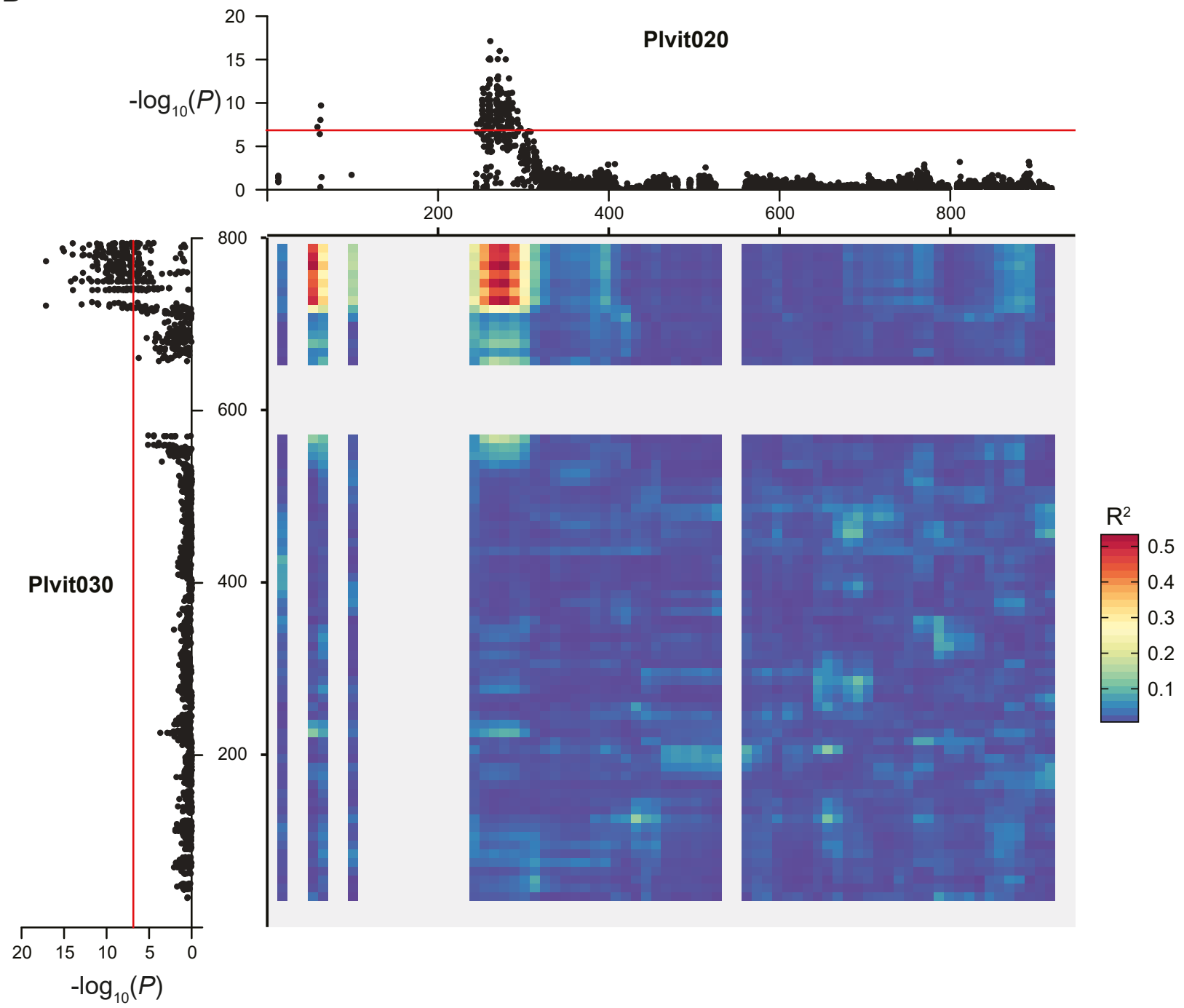

Figure 1 Genome-wide association analysis for identifying mating-type regions in Plasmopara viticola. A: Manhattan plot of the negative $\log _{10}$-transformed association $P$-values between mating type and SNPs along the $P l$. viticola genome. Alternating black and grey blocks of dots mark the limits between scaffolds. The two scaffolds with a significant association signal (Plvit020 and Plvit030) are indicated with arrows. Only 5\% of the SNPs with a $P$ value $<0.1$ are represented, to keep the number of plotted points manageable. B: Manhattan plots of the negative $\log 10$-transformed association $P$-values between mating type and SNPs for Plvit020 and Plvit030, and linkage disequilibrium between the two scaffolds represented as a heatmap ( 80 bins). The significance threshold for the association analysis, computed with 10,000 permutations, is represented as a red line in both panels. 
region probably lacked recombination. The rate of $\mathrm{LD}$ decay was much lower in this region than in the rest of the genome (Supplementary Figure 2), providing further support for the hypothesis of a lack of recombination. The incomplete assembly of this locus in the reference genome was probably due to its high repeat content. Indeed, we observed two large regions composed exclusively of tandem repeat arrays covering 327 kb (Supplementary Figure 3).

We therefore used a reference-free SNP calling method to identify potential missing sequences between the two scaffolds. We detected short genomic sequences carrying SNPs with significant signals of association with the mating-type phenotype that were missing from the reference assembly (see Material \& Methods section). We reassembled the PacBio reads corresponding to these short sequences and obtained a new contig, Plvit600 (32,220 bp), with SNPs significantly associated with the mating-type phenotype in strong linkage disequilibrium with SNPs located at the edges of Plvit020 and Plvit030, and therefore probably located between these two scaffolds (Supplementary Figure 4). Plvit600 also included a large region entirely composed of tandem repeat arrays (Supplementary Figure 3). We therefore concluded that the mating-type locus was at least $570 \mathrm{~kb}$ long (Figure 2). P2 individuals were homozygous for the reference allele (designated MAT-a) at the mating-type locus, whereas P1 individuals were heterozygous, carrying the MAT-a reference allele and a second allele, called MAT-b (Figure 3). We found no homozygous individual for the alternative MAT-b allele. This suggested that the MAT-b allele is dominant. The two alleles were highly differentiated along the $570 \mathrm{~kb}$, as shown by the large difference of heterozygosity levels (Figure 2), again consistent with a lack of recombination in this region.

The mating-type region included a total of 40 predicted coding sequences (Supplementary Table 3), 26 of which had predicted functions and did not correspond to TEs (Table 1). Based on the predicted functions of these genes, the most promising candidate for involvement in mating- 


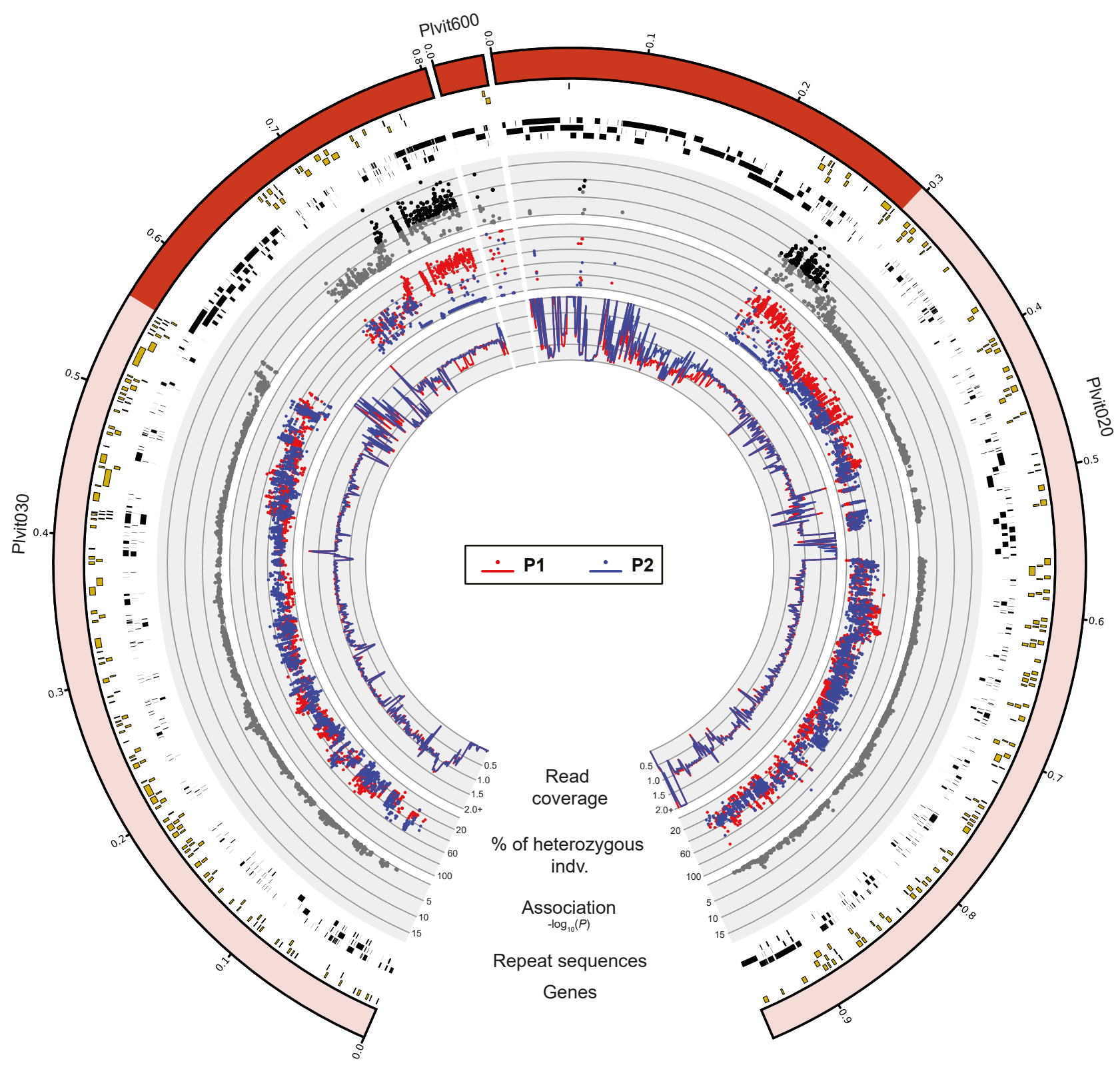

Figure 2 Genomic regions associated with mating type in Plasmopara viticola. The regions significantly associated with the mating-type phenotype are shown in dark red within the scaffold ideograms (outer track). Annotated genes are represented as yellow rectangles, and repeated sequences as black rectangles. Significant negative $\log _{10}$-transformed $P$-values from the association analysis are represented by black dots, and other values are represented by gray dots. The percentage of heterozygous individuals for each SNP is shown for individuals of mating types P1 by red dots and P2 by blue dots. The mean normalized read coverage (for adjacent $1 \mathrm{~kb}$ windows) is shown by red lines for P1 individuals and blue lines for P2 individuals. The data were obtained with the referencebased SNP calling approach for the scaffolds Plvit020 and Plvit030 and the reference-free SNP calling approach for Plvit600. 


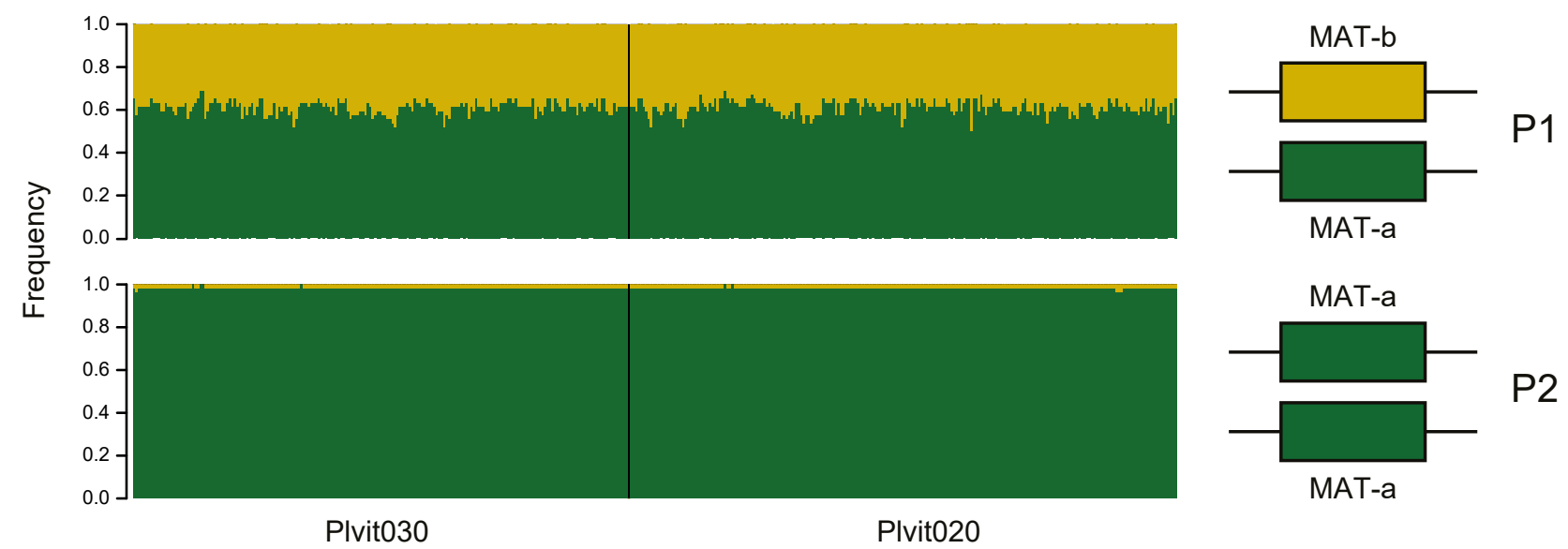

Figure 3 Mating type is determined by a two-allele system in Plasmopara viticola. Left panel: Allele frequencies at SNPs associated with the mating-type phenotype in P1 individuals (top) and P2 individuals (bottom). Only results for the reference-based SNP calling approach are shown. Right panel: Proposed model for mating-type determination, with homozygosity in P2 individuals and heterozygosity in P1 individuals. For both panels, the reference allele (i.e. the allele found in the reference genome) is shown in green, and the alternative allele is shown in yellow.

type determination was a gene encoding a transmembrane protein (PVIT_0010929.T1) with a sterol-sensing domain and lipid transport activity. This protein might act as a hormone receptor, and hormones have been identified as mating-type factors during initiation of sexual reproduction in Phytophthora spp. (Qi et al. 2005; Harutyunyan et al. 2008; Ojika et al. 2011). Another two genes (PVIT_0010925.T1 and PVIT_0010927.T1) encoded proteins with lipid-binding domains that could potentially interact with mating hormones. We also found a putative mitogen-activated protein (MAP) kinase gene (PVIT_0027771.T1) in the identified mating-type region. MAP kinases play an important role in the mating pheromone response pathway in fungi (Zhao et al. 2007), and genes encoding kinases are present at the mating-type loci of some fungi (Karos et al. 2000). We also found genes encoding a protein with an HRCD (helicase and RNaseD C-terminal; PVIT_0010933.T1) domain and a RecQ-mediated genome instability protein 2 (PVIT_0008617.T1). Proteins of this type can interact with DNA and are often associated with RecQ helicases and, thus, DNA repair. These proteins may also be involved in recombination 
Table 1 Putative function of genes in the mating-type locus of Plasmopara viticola

\begin{tabular}{|c|c|c|c|c|}
\hline Protein ID & Scaffold & Length & Putative function & $\begin{array}{c}\text { Non-syn. } \\
\text { mutations* }\end{array}$ \\
\hline PVIT_0008600.T1 & Plvit020 & 795 & transferase activity, transferring acyl groups & \\
\hline PVIT_0008605.T1 & Plvit020 & 752 & transferase activity, transferring acyl groups & \\
\hline PVIT_0008608.T1 & Plvit020 & 713 & transferase activity, transferring acyl groups & I234T, Y239C \\
\hline PVIT_0008616.T1 & Plvit020 & 1375 & $\begin{array}{l}\text { similar to peroxin-1, involved in protein targeting to } \\
\text { peroxisome. Contains a SEP and an UBX domains. }\end{array}$ & $\begin{array}{l}\text { P179S, D392G, } \\
\text { E540D, R763K }\end{array}$ \\
\hline PVIT_0008617.T1 & Plvit020 & 160 & similar to RecQ-mediated genome instability protein 2 & \\
\hline PVIT_0008618.T1 & Plvit020 & 363 & $\begin{array}{l}\text { disulphide isomerase, involved in cell redox homeostasis } \\
\text { and protein folding in the endoplasmic reticulum }\end{array}$ & \\
\hline PVIT_0008619.T1 & Plvit020 & 83 & molybdopterin synthase sulfur carrier subunit & \\
\hline PVIT_0008620.T1 & Plvit020 & 303 & similar to peroxin-13 & $\begin{array}{l}\text { V79G, L233S, } \\
\text { E237D }\end{array}$ \\
\hline PVIT_0010914.T1 & Plvit030 & 419 & Glycosyltransferase GlcNAc & \\
\hline PVIT_0010915.T1 & Plvit030 & 270 & protein N-terminal amidase, similar to NTA1 & \\
\hline PVIT_0010916.T1 & Plvit030 & 218 & $\begin{array}{c}\text { similar to sentrine/SUMO-specific protease } 8 \text { (SENP8). } \\
\text { Contains an Ulp1 catalytic domain }\end{array}$ & \\
\hline PVIT_0010917.T1 & Plvit030 & 449 & $\begin{array}{l}\text { similar to } 26 \mathrm{~S} \text { proteasome regulatory subunit N13. } \\
\text { Contains an ubiquitin interacting motif }\end{array}$ & \\
\hline PVIT_0010918.T1 & Plvit030 & 765 & cation efflux protein / zinc transporter & \\
\hline PVIT_0010919.T1 & Plvit030 & 137 & dynein light chain roadblock-type & \\
\hline PVIT_0010922.T1 & Plvit030 & 203 & $\begin{array}{c}\text { similar to protein N-lysine methyltransferase } \\
\text { METTL21A }\end{array}$ & \\
\hline PVIT_0010924.T1 & Plvit030 & 388 & thiol-dependent ubiquitin-specific protease activity & \\
\hline PVIT_0010925.T1 & Plvit030 & 1071 & $\begin{array}{l}\text { FYVE-finger-containing Rab5 effector protein } \\
\text { Rabenosyn-5-related. Contains a START-like domain }\end{array}$ & \\
\hline PVIT_0010926.T1 & Plvit030 & 993 & calcium binding protein & \\
\hline PVIT_0010927.T1 & Plvit030 & 1230 & $\begin{array}{l}\text { Bactericidal permeability-increasing protein. Lipid \& } \\
\text { protein binding. }\end{array}$ & \\
\hline PVIT_0010928.T1 & Plvit030 & 633 & $\begin{array}{l}\text { similar to DNA polymerase eta. Contains a DNA } \\
\text { polymerase, Y-family, little finger domain. DNA repair }\end{array}$ & H163Y \\
\hline PVIT_0010929.T1 & Plvit030 & 1479 & $\begin{array}{c}\text { transmembrane protein with sterol-sensing domain. } \\
\text { Involved in lipid transport, translational initiation, cell } \\
\text { division }\end{array}$ & $\begin{array}{l}\text { I297M, L402M, } \\
\text { E1211D }\end{array}$ \\
\hline PVIT_0010931.T1 & Plvit030 & 417 & similar to serine / arginine rich splicing factor & $\begin{array}{c}\text { D229G, L284R, } \\
\text { S294G, S303G, } \\
\text { T306S, G310S, } \\
\text { G325V }\end{array}$ \\
\hline PVIT_0010932.T1 & Plvit030 & 390 & ADP-ribosylation factor-like 2-binding protein domain & $\begin{array}{l}\text { G3D, L5F, S52T, } \\
\text { H159Q, K219E, } \\
\text { premature start }\end{array}$ \\
\hline PVIT_0010933.T1 & Plvit030 & 479 & Contains an HRDC domain & $\begin{array}{l}\text { D130N, K176R, } \\
\text { L165I, V249I, } \\
\text { T250S }\end{array}$ \\
\hline PVIT_0027771.T1 & Plvit600 & 444 & Mitogen-activated protein kinase & \\
\hline PVIT_0027772.T1 & Plvit600 & 842 & $\begin{array}{l}\text { similar to transitional endoplasmic reticulum ATPase } \\
\text { CDC48 }\end{array}$ & \\
\hline
\end{tabular}

*Non-synonymous mutations caused by alternate alleles (i.e. present in the MAT-b allele) for SNPs associated with the matingtype phenotype. 
suppression or gene regulation. This is potentially also the case for a Cdc48-like protein (PVIT_0027772.T1) encoded by a gene present at the mating-type locus. Cdc48 can interact with protein complexes on chromatin and is involved in gene regulation and cell cycle progression. Finally, we also found four genes (PVIT_0008616.T1, PVIT_0010916.T1, PVIT_0010917.T1 and PVIT_0010924.T1) encoding proteins with ubiquitin-like or ubiquitin-interacting domains, which could potentially be involved in addressing proteins to the proteasome and, thus, in protein degradation.

Using a reciprocal best hit approach with annotated whole proteomes, we identified the mating-type region in the genomes of Ph. infestans, and two other species more closely related to Pl. viticola: Br. lactucae and Pl. halstedii. The genes orthologous to putative mating-type genes were found on three scaffolds for Ph. infestans, one for $\mathrm{Br}$. lactucae and two for $\mathrm{Pl}$. halstedii (Figure 4, Supplementary Table 4). The genomic region including the candidate genes differed in size between species, probably reflecting the difference in repeat content between the species genomes (Haas et al. 2009; Pecrix et al. 2019; Dussert et al. 2019). We observed differences of gene order between species, suggesting the existence of genomic rearrangements of the matingtype locus between species, as expected for non-recombining mating-type regions (Badouin et al. 2015).

\section{DISCUSSION}

We report here the first identification of an oomycete mating-type locus sequence, in the genome of $\mathrm{Pl}$. viticola. The $\mathrm{Pl}$. viticola mating-type locus was found in a $570 \mathrm{~kb}$ nonrecombining, repeat-rich region of the genome encompassing 40 genes. The region was heterozygous (MAT-a/MAT-b) in the P1 mating type and homozygous (MAT-a/MAT-a) in the P2 mating type, indicating dominance of the MAT-b allele. These findings are consistent with 
A

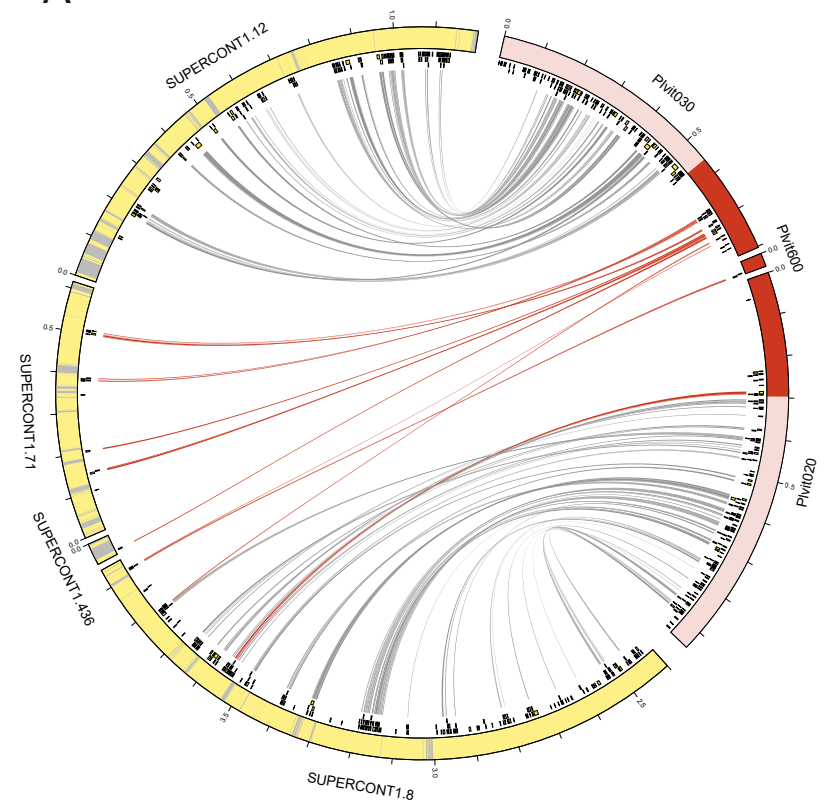

vs. Phytophthora infestans
B

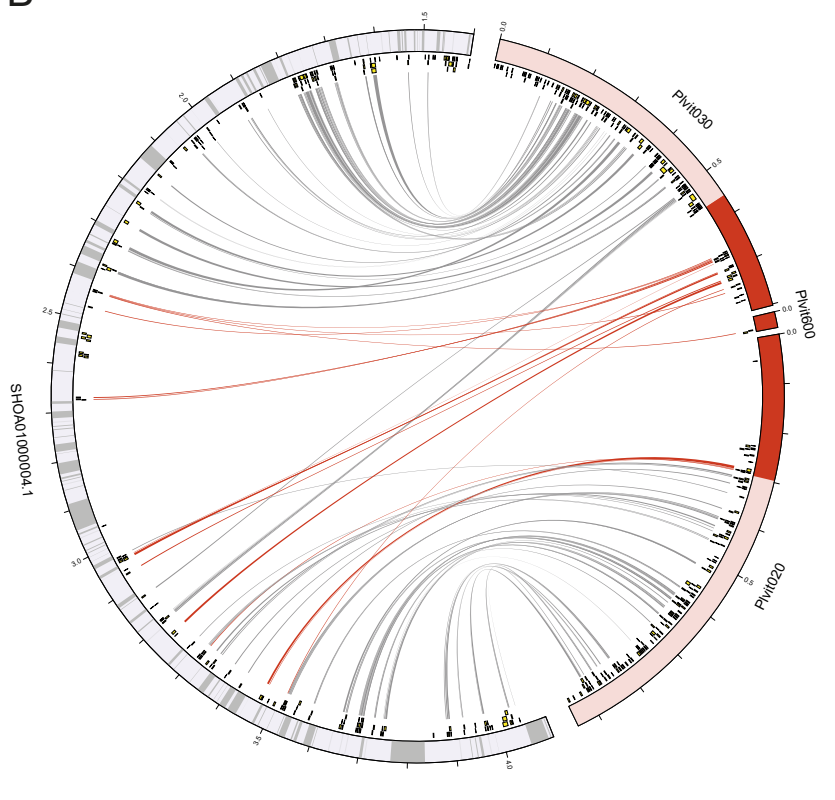

vs. Bremia lactucae

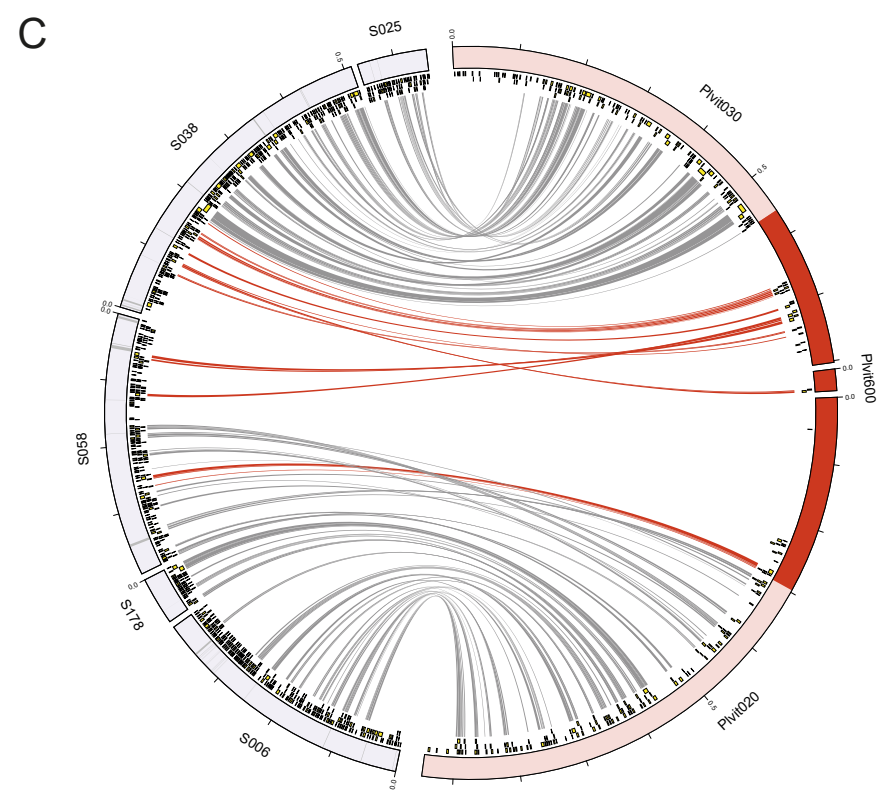

vs. Plasmopara halstedii

Figure 4 Orthologs of the Plasmopara viticola mating-type locus genes in the genomes of Phytophthora infestans (A), Bremia lactucae (B) and Plasmopara halstedii (C). Orthology relationships were determined with reciprocal best hits and are represented by links between ideograms. The regions significantly associated with the mating-type phenotype in $\mathrm{Pl}$. viticola and the links corresponding to genes in these regions are shown in dark red. Predicted genes are represented by yellow boxes, and the grayed-out regions in ideograms represent gaps in the assemblies. 
previous findings for marker segregation analyses in Phytophthora species and Br. lactucae (Judelson 1996; Fabritius \& Judelson 1997; Sicard et al. 2003). This genetic determinism of mating type is unique in the tree of life and resembles determinism based on sex chromosomes, with one sex typically being homozygous and the other heterozygous. By contrast, fungal mating types are determined at the haploid stage, with mating occurring between gametes carrying different mating-type alleles, and many species harboring multiple alleles. Diploid or dikaryotic fungal individuals are necessarily heterozygous at the mating type locus in heterothallic species and can thus undergo selfing (Giraud et al. 2008). Mating types in fungi therefore do not prevent diploid selfing, they only prevent same-clone mating (Billiard et al. 2012), while mating types in oomycetes and plants do prevent diploid selfing. Self-incompatibility systems in plants are similar to oomycete mating types, as they prevent same-allele mating, and also display multiple alleles (Fujii et al. 2016).

As expected for non-recombining regions under long-term balancing selection, we found the two $P l$. viticola mating types to be highly divergent. The mating-type locus in Ph. infestans is also heteromorphic, with highly differentiated alleles, hemizygous fragments and genomic translocations, and it is associated with deleterious recessive alleles (Judelson 1996; Randall et al. 2003; Van Der Lee et al. 2004). The high degree of divergence between alleles, the number of rearrangements, the high repeat content, and the sheltering of deleterious alleles by permanent heterozygosity are typical of sex and mating-type chromosomes in plants, animals and fungi (Bachtrog 2005; Graves 2006; Badouin et al. 2015; Charlesworth 2015).

The localization of the mating-type genomic sequence and elucidation of the determinism of mating type (homozygous versus heterozygous) in an oomycete, and the identification of orthologous mating-type loci in other oomycete species will have fundamental implications for our understanding of the evolution of mating types in this lineage, and should shed light on why 
several other oomycetes have no self-incompatibility systems and can self, a state known as homothallism in oomycetes (Judelson 2009). In fungi, homothallic species often have the two mating-type alleles in each haploid genome (Butler 2014). Our study also paves the way for further investigations of the proximal molecular mechanisms determining mating type in oomycetes. We identified possible mating type-determining genes, including one encoding a putative hormone receptor. This may guide the development of innovative control methods based on disruption of the sexual cycle of the pathogen, since infections from oospores play a major role during grapevine downy mildew epidemics (Gobbin et al. 2005). Furthermore, the identification of the mating-type locus in such an economically important crop pathogen may improve our understanding of pathogen adaptation, as sex and outcrossing promote rapid evolution and resistance to harsh conditions.

\section{MATERIALS AND METHODS}

\section{Sampling and preparation of the study material}

Grapevine leaves infected with $P l$. viticola were collected across Europe (Supplementary Table 1). Diploid oomycete individuals were isolated from diseased leaves by dilution to isolate single spores, as follows: isolates of $\mathrm{Pl}$. viticola were first propagated on detached leaves of Vitis vinifera cv. Cabernet-Sauvignon. Sporangia were collected and suspended in water at a concentration of $10^{4}$ sporangia/mL (estimated using Malassez cells) and serial dilutions were performed to obtain a concentration of 0.1 sporangia/10 $\mu \mathrm{L}$. We applied $10 \mu \mathrm{L}$ of this inoculum to leaf disks, which were then placed in a growth chamber at $22^{\circ} \mathrm{C}(12 \mathrm{~h} \mathrm{light} / 12 \mathrm{~h}$ dark photoperiod) for seven days. We retained a single sporulating disk per isolate, which was, thus, derived from a single diploid sporangium. 


\section{DNA extraction and whole-genome resequencing}

Detached leaves from V. vinifera cv. Cabernet-Sauvignon or Muscat Ottonel plants were sterilized with bleach, washed with sterilized water and infected with fresh Pl. viticola inoculum. Leaves were placed in a growth chamber for $4-7$ days at $21-22^{\circ} \mathrm{C}(12 \mathrm{~h} / 12 \mathrm{~h}$ or $16 \mathrm{~h} / 8 \mathrm{~h}$ light/dark photoperiod). Sporangiophores and sporangia were then collected in distilled water. The suspension was centrifuged at maximum speed, the supernatant was removed and the pellet was stored at $-80{ }^{\circ} \mathrm{C}$. DNA was extracted with a modified CTAB procedure (Dussert et al. 2019) or with the Qiagen DNeasy Plant Mini Kit.

Genomic DNA was sequenced by Beckman Coulter Genomics (Grenoble, France) or at the GeT-PlaGe facility (Toulouse, France), on an Illumina HiSeq 2000 sequencer (2x100 bp paired-end reads) or an Illumina HiSeq 3000 sequencer (2x150 bp). Read lengths and mean genome coverage are provided in Supplementary Table 1.

\section{Mating-type determination}

Six Pl. viticola individuals (Supplementary Tables $1 \& 2$ ) were chosen as mating-type testers: three individuals of each mating type. These testers were first crossed with each other in all possible pairings, for separation of the six individuals into two opposite mating-type groups, P1 and P2 (three individuals each). We then crossed 54 individuals with these six testers to determine their mating type. In all experiments, leaf disks from $V$. vinifera cv. CabernetSauvignon plants were placed on $20 \mathrm{~g} / \mathrm{L}$ agar in Petri dishes and co-inoculated with $15 \mu \mathrm{L}$ droplets containing $4 \times 10^{4}$ sporangia/mL from each individual (three droplets per disk, six replicate disks per pairing). After four to five days in a growth chamber at $22^{\circ} \mathrm{C}(12 \mathrm{~h} \mathrm{light} / 12 \mathrm{~h}$ dark photoperiod), disks were examined for sporulation, to check for successful inoculation. The leaf disks were then transferred to a growth chamber at $12^{\circ} \mathrm{C}(12 \mathrm{~h} \mathrm{light} / 12 \mathrm{~h}$ dark photoperiod $)$ 
for about three weeks. Each leaf disk was then observed with a binocular microscope, to check for the presence of oospores, indicative of successful mating.

\section{Read mapping and single-nucleotide polymorphism (SNP) calling}

Reads from each individual were mapped onto the $P l$. viticola PacBio reference genome (Dussert et al. 2019) with the Glint aligner v1.0.rc12.826:833 (available at https://forgedga.jouy.inra.fr/projects/glint), allowing a maximum of $15 \%$ mismatches, to handle genomic regions with high heterozygosity (other parameters: mappe mode, --no-lc-filtering --best-score -lrmin 80), and filtering out reads with a mapping quality below 3 and reads that were not properly paired.

A pileup file was generated from the resulting alignment files with SAMtools 1.3.1 (Li et al. 2009) mpileup without probabilistic alignment quality computation (-B parameter). SNPs and short insertions and deletions (indels) were then called with the multi-sample procedure (mpileup2snp and mpileup2indel) of VARSCAN 2.4.3 (Koboldt et al. 2012) (--min-coverage 10 -min-reads2 5 --min-avg-qual 15 --min-var-freq 0.2 --min-freq-for-hom 0.75 --p-value 0.001 -strand-filter 1). For each individual, sites with a read coverage more than 1.5 standard deviations on either side of the mean value for genome coverage were discarded with BCFtools 1.1-60g3d5d3d9 (Li 2011). SNPs located within 2 bp of a detected indel were also filtered out. Finally, only sites with less than $10 \%$ missing data were retained. After filtering, the dataset contained 2,011 million SNPs.

\section{Genetic diversity and association of SNPs with mating types}

We used SAMtools to assess the normalized read coverage for $1 \mathrm{~kb}$ windows for each individual. Using only SNPs with a minor allele frequency (MAF) below 0.1, the percentage of 
heterozygous individuals for each site along the genome was calculated separately for P1 and P2 individuals with VCFtools (Danecek et al. 2011). Linkage disequilibrium between sites, as assessed with the $\mathrm{R}^{2}$ statistic, was also computed with VCFtools. Finally, linkage disequilibrium decay along scaffolds was analyzed with PopLDdecay 3.28 (Zhang et al. 2019).

SNP thinning was performed by clumping (as described in Privé et al. 2018) with PLINK 1.07 (Purcell et al. 2007), with MAF as the statistic of importance and a LD threshold ( $\mathrm{R}^{2}$ ) of 0.2. Population genetic structure was investigated by principal component analysis (PCA) with PCAdapt (Luu et al. 2017) in R (R Core Team 2017). We also investigated population clustering by computing ancestry coefficients for individuals (Q-matrix) with the non-negative matrix factorization algorithm sNMF (Frichot et al. 2014) implemented in the LEA package (Frichot \& François 2015) in R. We set the number of groups $K$ between 1 and 10, with 50 runs for each value of $K$. The optimal number of groups was chosen using the minimum cross-entropy criterion.

For identification of the genomic region controlling mating types, we analyzed the association between genotype and mating type (genome-wide association approach) with TASSEL 5.2.41 (Bradbury et al. 2007) on SNPs with no missing data and a MAF above 0.1, using a GLM model with the Q-matrix estimated by sNMF. $P$-values were corrected with 10,000 permutations (threshold: 0.05). We filtered out the small number of association signals found in short scaffolds, corresponding to isolated SNPs in repeat regions.

\section{Identification of genomic regions associated with mating type and absent from the reference}

\section{genome}

The discovery of SNPs associated with mating type in the edges of two scaffolds suggested that the mating-type locus had been only partially assembled in the reference assembly. 
We therefore used discoSnp++ (Peterlongo et al. 2017), a reference-free method for identifying SNPs. Briefly, SNPs are detected from bubbles found in a de Bruijn graph built from raw sequencing reads, and short contigs are reconstructed around the SNPs. This analysis was carried out with a subset of 30 individuals (Supplementary Table 1), as computing resource issues arose if the analysis was extended to a larger number of individuals. We used a $k$-mer size of 31 , the smart branching strategy (-b 1), a maximum of five SNPs in unique bubbles (-P 5), and polymorphisms were extended with left and right contigs (-T). Only sites with less than $10 \%$ missing data and a MAF below 0.1 were retained. Contigs were mapped onto the reference genome with the VCF_creator module of discoSnp++ and an association analysis was carried out with TASSEL, as described above, to check that our results were robust to the SNP calling method.

Contigs from discoSnp++ including SNPs significantly associated with mating type were aligned with the self-corrected PacBio reads used for reference genome assembly (Dussert et al. 2019) with BLASTN+, retaining hits displaying at least $95 \%$ identity and $95 \%$ query coverage. PacBio reads corresponding to these hits were assembled with SMARTdenovo (available from https://github.com/ruanjue/smartdenovo) using default parameters. Assembled contigs were aligned against the reference scaffolds including SNPs with a significant association signal, with NUCmer from the MUMmer 3.23 package (Kurtz et al. 2004) using default parameters, and only regions absent from the reference assembly were retained. Available paired-end reads and $3 \mathrm{~kb}$ mate-pair reads (Dussert et al. 2016) and the self-corrected PacBio reads were aligned against the reference genome and the new mating-type contigs, with BWA 0.7.12 (Li \& Durbin 2009) for short reads and NGMLR (Sedlazeck et al. 2018) for long reads. Aligned reads were used to polish the new mating-type contigs with Pilon 1.22 (Walker et al. 2014). 
After sequence polishing, contigs from the discoSNP analysis were again mapped, but this time to the reference assembly and the newly assembled contigs, and a final association analysis was carried out. Newly assembled contigs with no SNP significantly associated with the matin type were discarded. For the remaining contigs, repeat sequences were annotated with TEannot from REPET 2.2 (Quesneville et al. 2005; Flutre et al. 2011), using the Pl. viticola repeat library (Dussert et al. 2019). Available RNA-seq data (Mestre et al. 2016; Dussert et al. 2019) were aligned against the reference assembly and the new contigs with STAR 2.4 (Dobin $e t$ al. 2013), and were used as hints for gene prediction in the new contigs with AUGUSTUS 3.1 (Stanke et al. 2008), together with the training files produced by the previous annotation (Dussert et al. 2019). Proteins for which functional domains of transposable elements were detected with InterProScan 5 (Jones et al. 2014) or for which hits were obtained with TransposonPSI (http://transposonpsi.sourceforge.net; 30\% coverage, e-value $<1 \mathrm{e}^{-10}$ ) were filtered out. The remaining proteins were aligned against the NCBI nr database with BLASTP+ (-evalue $1 \mathrm{e}^{-5}$ max_hsp 20 -num_alignment 20), and functionally annotated with Blast2GO 4.1.9 (Gotz et al. 2008) using the BLASTP+ and InterProScan results for gene ontology (GO) term mapping.

For all candidate genes within the mating-type locus (i.e. from the scaffolds in the reference assembly and the new contigs), we further checked whether coding sequences corresponded to transposable elements: protein sequences for those genes were masked with CENSOR 4.2.29 (Kohany et al. 2006) using RepBase 23.08, which allowed us to compute for each protein a percentage of coverage from known transposable elements.

\section{Comparative genomics with Phytophthora infestans, Bremia lactucae and Plasmopara}

\section{halstedii}


We identified the orthologs of the mating-type candidate genes in the annotated proteomes of Ph. infestans (Haas et al. 2009, ASM14294v1 assembly), Br. lactucae (Fletcher et al. 2019) and Pl. halstedii (Pecrix et al. 2019), with the reciprocal best hits (RBH) method using BLASTP+ (-evalue $1 \mathrm{e}^{-6}$-qcov_hsp_perc 50 -use_sw_tback, min. identity 40\%). Results were visualized with Circos (Krzywinski et al. 2009).

\section{DATA AVAIBILITY}

Raw reads for each individual will be deposited in GenBank (see Supplementary Table 1 for SRA accession numbers) and analysis files have been deposited in Dataverse (doi.org/10.15454/ILQ12T).

\section{ACKNOWLEDGMENTS}

We thank Vincent Thomas for helping with the production of material and Pal Kozma (University of Pécs, Hungary), Mauro Jermini (Agroscope, Switzerland), Sara Legler (UCSC, Italy) Hervé Steva (Bordeaux, France) and Atanas Atanassov (JGC, Bulgaria) for providing $P l$. viticola isolates. We are grateful to Jérôme Gouzy (INRAE, Toulouse) for his help and suggestions concerning data analysis. We thank the Genotoul bioinformatics platform Toulouse Midi-Pyrenees (Bioinfo Genotoul) for providing help and computing resources. This work was performed in collaboration with the GeT core facility, Toulouse, France (http://get.genotoul.fr). This study was supported by the European Commission (INNOVINE, FP7-KBBE-311775), and the French National Research Agency (GANDALF project, ANR-12-ADAP-0009; EFFECTOORES project, ANR-13-ADAP-0003; Investments for the future program in the Cluster of Excellence COTE, ANR-10-LABX-45; Investments for the future program France 
Génomique National infrastructure, ANR-10-INBS-09). TG acknowledges receipt of the ERC advanced grant EvolSexChrom \#832252.

\section{REFERENCES}

Bachtrog D (2005) Sex chromosome evolution: Molecular aspects of Y-chromosome degeneration in Drosophila. Genome Research, 15, 1393-1401.

Badouin H, Hood ME, Gouzy J, et al. (2015) Chaos of rearrangements in the mating-type chromosomes of the anther-smut fungus Microbotryum lychnidis-dioicae. Genetics, 200, 1275-1284.

Beukeboom LW, Perrin N (2014) The evolution of sex determination. Oxford University Press, USA.

Billiard S, López-Villavicencio M, Hood ME, Giraud T (2012) Sex, outcrossing and mating types: unsolved questions in fungi and beyond. Journal of Evolutionary Biology, 25, 1020-1038.

Bradbury PJ, Zhang Z, Kroon DE, et al. (2007) TASSEL: software for association mapping of complex traits in diverse samples. Bioinformatics, 23, 2633-2635.

Brurberg MB, Elameen A, Le VH, et al. (2011) Genetic analysis of Phytophthora infestans populations in the Nordic European countries reveals high genetic variability. Fungal Biology, 115, 335-342.

Butler G (2014) The Evolution of MAT: The Ascomycetes. In: Sex in Fungi (eds Heitman J, Kronstad J, Taylor J, Casselton L), pp. 3-18. ASM P, Washington, DC.

Charlesworth D (2015) Plant contributions to our understanding of sex chromosome evolution. New Phytologist, 208, 52-65.

Charlesworth D, Charlesworth B (1987) Inbreeding depression and its evolutionary consequences. Annual Review of Ecology and Systematics, 18, 237-268.

Charlesworth D, Morgan MT, Charlesworth B (1990) Inbreeding depression, genetic load, and the evolution of outcrossing rates in a multilocus system with no linkage. Evolution, 44, 1469-1489.

Danecek P, Auton A, Abecasis G, et al. (2011) The variant call format and VCFtools. Bioinformatics, 27, 21562158.

Delmas CEL, Dussert Y, Delière L, et al. (2017) Soft selective sweeps in fungicide resistance evolution: recurrent mutations without fitness costs in grapevine downy mildew. Molecular Ecology, 26, 1936-1951.

Delmas CEL, Fabre F, Jolivet J, et al. (2016) Adaptation of a plant pathogen to partial host resistance: selection for greater aggressiveness in grapevine downy mildew. Evolutionary Applications, 9, 709-725.

Dobin A, Davis CA, Schlesinger F, et al. (2013) STAR: ultrafast universal RNA-seq aligner. Bioinformatics, 29, 1521.

Dussert Y, Gouzy J, Richart-Cervera S, et al. (2016) Draft genome sequence of Plasmopara viticola, the grapevine downy mildew pathogen. Genome Announcements, 4, e00987-16.

Dussert Y, Mazet ID, Couture C, et al. (2019) A high-quality grapevine downy mildew genome assembly reveals rapidly evolving and lineage-specific putative host adaptation genes. Genome Biology and Evolution, 11, 954969.

Fabritius AL, Judelson HS (1997) Mating type loci segregate aberrantly in Phytophthora infestans but normally in Phytophthora parasitica: Implications for models of mating type determination. Current Genetics, 32, 60-65.

Fletcher K, Gil J, Bertier LD, et al. (2019) Genomic signatures of heterokaryosis in the oomycete pathogen Bremia lactucae. Nature Communications, $\mathbf{1 0 .}$

Flutre T, Duprat E, Feuillet C, Quesneville H, Barton G (2011) Considering transposable element diversification in de novo annotation approaches. PLoS ONE, $\mathbf{6}$, e16526.

Fontaine MC, Austerlitz F, Giraud T, et al. (2013) Genetic signature of a range expansion and leap-frog event after the recent invasion of Europe by the grapevine downy mildew pathogen Plasmopara viticola. Molecular Ecology, 22, 2771-2786.

Frichot E, François O (2015) LEA : An R package for landscape and ecological association studies. Methods in 
Ecology and Evolution, 6, 925-929.

Frichot E, Mathieu F, Trouillon T, Bouchard G, François O (2014) Fast and efficient estimation of individual ancestry coefficients. Genetics, 196, 973-83.

Fujii S, Kubo KI, Takayama S (2016) Non-self- and self-recognition models in plant self-incompatibility. Nature Plants, 2.

Gavino PD, Smart CD, Sandrock RW, et al. (2000) Implications of sexual reproduction for Phytophthora infestans in the United States: Generation of an aggressive lineage. Plant Disease, 84, 731-735.

Giraud T, Yockteng R, López-Villavicencio M, Refrégier G, Hood ME (2008) Mating system of the anther smut fungus Microbotryum violaceum: Selfing under heterothallism. Eukaryotic Cell, 7, 765-775.

Gobbin D, Jermini M, Loskill B, et al. (2005) Importance of secondary inoculum of Plasmopara viticola to epidemics of grapevine downy mildew. Plant Pathology, 54, 522-534.

Gotz S, Garcia-Gomez JM, Terol J, et al. (2008) High-throughput functional annotation and data mining with the Blast2GO suite. Nucleic Acids Research, 36, 3420-3435.

Graves JAM (2006) Sex chromosome specialization and degeneration in mammals. Cell, 124, 901-914.

Haas BJ, Kamoun S, Zody MC, et al. (2009) Genome sequence and analysis of the Irish potato famine pathogen Phytophthora infestans. Nature, 461, 393-398.

Harutyunyan SR, Zhao Z, Den Hartog T, et al. (2008) Biologically active Phytophthora mating hormone prepared by catalytic asymmetric total synthesis. Proceedings of the National Academy of Sciences, 105, 8507-8512.

Igic B, Lande R, Kohn JR (2008) Loss of self-incompatibility and its evolutionary consequences. International Journal of Plant Sciences, 169, 93-104.

Jones P, Binns D, Chang H-Y, et al. (2014) InterProScan 5: genome-scale protein function classification. Bioinformatics, 30, 1236-1240.

Judelson HS (1996) Chromosomal heteromorphism linked to the mating type locus of the oomycete Phytophthora infestans. Molecular and General Genetics, 252, 155-161.

Judelson HS (2009) Sexual reproduction in Oomycetes: biology, diversity and contributions to fitness. In: Oomycete genetics and genomics: diversity, interactions and research tools (eds Lamour K, Kamoun S), pp. 121-138. John Wiley \& Sons, Inc., Hoboken, New Jersey.

Judelson HS, Spielman LJ, Shattock RC (1995) Genetic mapping and non-Mendelian segregation of mating type loci in the oomycete, Phytophthora infestans. Genetics, 141.

Karos M, Chang YC, McClelland CM, et al. (2000) Mapping of the Cryptococcus neoformans MATa locus: Presence of mating type-specific mitogen-activated protein kinase cascade homologs. Journal of Bacteriology, 182, 6222-6227.

Koboldt DC, Zhang Q, Larson DE, et al. (2012) VarScan 2: somatic mutation and copy number alteration discovery in cancer by exome sequencing. Genome Research, 22, 568-76.

Kohany O, Gentles AJ, Hankus L, Jurka J (2006) Annotation, submission and screening of repetitive elements in Repbase: RepbaseSubmitter and Censor. BMC Bioinformatics, 7, 474.

Krzywinski M, Schein J, Birol I, et al. (2009) Circos: an information aesthetic for comparative genomics. Genome Research, 19, 1639-45.

Kurtz S, Phillippy A, Delcher AL, et al. (2004) Versatile and open software for comparing large genomes. Genome Biology, 5, R12.

Lande R, Porcher E (2015) Maintenance of quantitative genetic variance under partial self-fertilization, with implications for evolution of selfing. Genetics, 200, 891-906.

Lande R, Schemske DW (1985) The evolution of self-fertilization and inbreeding depression in plants. I. Genetic models. Evolution, 39, 24-40.

Van Der Lee T, Testa A, Robold A, Van't Klooster J, Govers F (2004) High-density genetic linkage maps of Phytophthora infestans reveal trisomic progeny and chromosomal rearrangements. Genetics, 167, 1643-1661.

Lévesque CA (2011) Fifty years of oomycetes-from consolidation to evolutionary and genomic exploration. Fungal Diversity, 50, 35-46.

Li H (2011) A statistical framework for SNP calling, mutation discovery, association mapping and population 
genetical parameter estimation from sequencing data. Bioinformatics, 27, 2987-2993.

Li H, Durbin R (2009) Fast and accurate short read alignment with Burrows-Wheeler transform. Bioinformatics, 25, 1754-1760.

Li H, Handsaker B, Wysoker A, et al. (2009) The sequence alignment/map format and SAMtools. Bioinformatics, 25, 2078-2079.

Luu K, Bazin E, Blum MGB (2017) pcadapt : an R package to perform genome scans for selection based on principal component analysis. Molecular Ecology Resources, 17, 67-77.

Mestre P, Carrere S, Gouzy J, et al. (2016) Comparative analysis of expressed CRN and RXLR effectors from two Plasmopara species causing grapevine and sunflower downy mildew. Plant Pathology, 65, 767-781.

Ojika M, Molli SD, Kanazawa H, et al. (2011) The second Phytophthora mating hormone defines interspecies biosynthetic crosstalk. Nature Chemical Biology, 7, 591-593.

Orias E, Singh DP, Meyer E (2017) Genetics and epigenetics of mating type determination in Paramecium and Tetrahymena. Annual Review of Microbiology, 71, 133-156.

Pecrix Y, Buendia L, Penouilh-Suzette C, et al. (2019) Sunflower resistance to multiple downy mildew pathotypes revealed by recognition of conserved effectors of the oomycete Plasmopara halstedii. The Plant Journal, 97, 730-748.

Peressotti E, Wiedemann-Merdinoglu S, Delmotte F, et al. (2010) Breakdown of resistance to grapevine downy mildew upon limited deployment of a resistant variety. BMC Plant Biology, 10, 147.

Peterlongo P, Riou C, Drezen E, Lemaitre C (2017) DiscoSnp++: de novo detection of small variants from raw unassembled read set(s). bioRxiv, 209965.

Privé F, Aschard H, Ziyatdinov A, Blum MGB (2018) Efficient analysis of large-scale genome-wide data with two R packages: bigstatsr and bigsnpr. Bioinformatics.

Purcell S, Neale B, Todd-Brown K, et al. (2007) PLINK: A tool set for whole-genome association and populationbased linkage analyses. The American Journal of Human Genetics, 81, 559-575.

Qi J, Asano T, Jinno M, et al. (2005) Characterization of a Phytophthora mating hormone. Science, 309, $1828-1828$.

Quesneville H, Bergman CM, Andrieu O, et al. (2005) Combined evidence annotation of transposable elements in genome sequences. PLoS Computational Biology, 1, e22.

R Core Team (2017) R: A language and environment for statistical computing. R Foundation for Statistical Computing, Vienna, Austria. URL: http://www.R-project.org/.

Randall TA, Ah Fong A, Judelson HS (2003) Chromosomal heteromorphism and an apparent translocation detected using a BAC contig spanning the mating type locus of Phytophthora infestans. Fungal Genetics and Biology, 38, 75-84.

Sedlazeck FJ, Rescheneder P, Smolka M, et al. (2018) Accurate detection of complex structural variations using single-molecule sequencing. Nature Methods, 15, 461-468.

Sekimoto H (2017) Sexual reproduction and sex determination in green algae. Journal of Plant Research, 130, 423431.

Sicard D, Legg E, Brown S, et al. (2003) A genetic map of the lettuce downy mildew pathogen, Bremia lactucae, constructed from molecular markers and avirulence genes. Fungal Genetics and Biology, 39, 16-30.

Stanke M, Diekhans M, Baertsch R, Haussler D (2008) Using native and syntenically mapped cDNA alignments to improve de novo gene finding. Bioinformatics, 24, 637-644.

Toffolatti SL, Prandato M, Serrati L, et al. (2011) Evolution of Qol resistance in Plasmopara viticola oospores. European Journal of Plant Pathology, 129, 331-338.

Vercesi A, Toffolatti SL, Zocchi G, Guglielmann R, Ironi L (2010) A new approach to modelling the dynamics of oospore germination in Plasmopara viticola. European Journal of Plant Pathology, 128, 113-126.

Walker BJ, Abeel T, Shea T, et al. (2014) Pilon: an integrated tool for comprehensive microbial variant detection and genome assembly improvement. PLOS ONE, 9, e112963.

van West P (2006) Saprolegnia parasitica, an oomycete pathogen with a fishy appetite: new challenges for an old problem. Mycologist, 20, 99-104.

Wong FP, Burr HN, Wilcox WF (2001) Heterothallism in Plasmopara viticola. Plant Pathology, 50, 427-432. 
bioRxiv preprint doi: https://doi.org/10.1101/2020.02.26.962936; this version posted February 27, 2020. The copyright holder for this preprint

(which was not certified by peer review) is the author/funder, who has granted bioRxiv a license to display the preprint in perpetuity. It is made available under aCC-BY-NC 4.0 International license.

Zhang C, Dong S-S, Xu J-Y, He W-M, Yang T-L (2019) PopLDdecay: a fast and effective tool for linkage disequilibrium decay analysis based on variant call format files. Bioinformatics, 35, 1786-1788.

Zhao X, Mehrabi R, Xu JR (2007) Mitogen-activated protein kinase pathways and fungal pathogenesis. Eukaryotic Cell, 6, 1701-1714. 